\title{
Misclassification probability as obese or lean in hypercaloric and normocaloric diet
}

\author{
ANDRÉ F. NASCIMENTO ${ }^{1}$, MÁRIO M. SUGIZAKI ${ }^{1}$, ANDRÉ S. LEOPOLDO ${ }^{1}$, \\ ANA P. LIMA-LEOPOLDO ${ }^{1}$, CÉLIA R. NOGUEIRA ${ }^{1}$, ETHEL L. B. NOVELLI ${ }^{3}$, \\ CARLOS R. PADOVANI ${ }^{2}$ and ANTONIO C. CICOGNA ${ }^{1}$
}

\author{
${ }^{1}$ Department of Medicine Clinical, Botucatu School of Medicine, São Paulo State University (UNESP) SP - \\ Brazil \\ ${ }^{2}$ Department of Biostatistics, Institute of Biological Science, São Paulo State University, Botucatu - São \\ Paulo - Brazil \\ ${ }^{3}$ Department of Chemistry and Biochemistry, Institute of Biological Science, São Paulo State University, \\ Botucatu - São Paulo - Brazil
}

\begin{abstract}
The aim of the present study was to determine the classification error probabilities, as lean or obese, in hypercaloric diet-induced obesity, which depends on the variable used to characterize animal obesity. In addition, the misclassification probabilities in animals submitted to normocaloric diet were also evaluated. Male Wistar rats were randomly distributed into two groups: normal diet (ND; $=31 ; 3,5 \mathrm{Kcal} / \mathrm{g}$ ) and hypercaloric diet (HD; $\mathrm{n}=31 ; 4,6 \mathrm{Kcal} / \mathrm{g}$ ). The ND group received commercial Labina rat feed and HD animals a cycle of five hypercaloric diets for a 14-week period. The variables analysed were body weight, body composition, body weight to length ratio, Lee index, body mass index and misclassification probability. A 5\% significance level was used. The hypercaloric pellet-diet cycle promoted increase of body weight, carcass fat, body weight to length ratio and Lee index. The total misclassification probabilities ranged from $19.21 \%$ to $40.91 \%$. In conclusion, the results of this experiment show that misclassification probabilities occur when dietary manipulation is used to promote obesity in animals. This misjudgement ranges from $19.49 \%$ to $40.52 \%$ in hypercaloric diet and $18.94 \%$ to $41.30 \%$ in normocaloric diet.
\end{abstract}

Key terms: Diet; diet-induced obesity; hypercaloric diet; misclassification, rats.

\section{INTRODUCTION}

Obesity is a complex multifactor disease characterized by the excessive accumulation of adipose tissue that may impair health. Nowadays, approximately 1.6 billion adults are overweight and at least 400 million adults are obese (WHO 2004). Even though genetic predisposition contributes to increase the number of obese individuals, the current world epidemic of obesity is a consequence of the enormous availability and consumption of highly energetic and palatable diets, and a reduction in energy use (Astrup et al. 1994; Lissner \& Heitmann 1995).
Since studies on the consequences of obesity in humans have ethical limitations, experimental models of diet-induced obesity are appropriate for studying this pathology, and many studies have been developed to obtain obese animals through diet changes (Sclafani \& Springer 1976; Ratting \& Clark 1984; Akiyama et al. 1996; Jang et al. 2003; Levin et al. 2003; Woods et al. 2003; Dourmashkin et al. 2005). Obesity in laboratory animals has been quantified by measuring visceral, epididymal and subcutaneous fat deposits (Woods et al. 2003; Thibault et al. 2004; Dourmashkin et al. 2005), total body fat (Levin et al. 2003; Woods et al. 2003;

Corresponding author: Tel: +55-14-38116213; Fax: +55-14-38116424. E-mail: nascimentoaf@yahoo.com.br (Antonio C. Cicogna). Address: School of Medicine, São Paulo State University, Distrito de Rubião Jr, s/n, CEP 18600-000, Botucatu SP- Brazil. 
Thibault et al. 2004), and body weight (Sclafani \& Springer 1976; Ratting \& Clark 1984; Akiyama et al. 1996; Jang et al. 2003; Woods et al. 2003). Woods et al. (2003) considered carcass fat as an obesity indicator and showed that using body weight led to underestimations in animal obesity levels. They demonstrated that, while the body weight of rats fed with a high-fat diet increased $10 \%$, the increase in carcass fat was between 30 and $50 \%$. This data suggests that the variables used to identify obese animals can present different responses and, therefore, lead to misclassification; for instance, animals submitted to high-fat diets may be classified as lean, in terms of body weight, even presenting characteristics of obese animal, in terms of carcass fat. The aim of the present study was to determine the classification error probabilities, as lean or obese, in hypercaloric diet-induced obesity, which depends on the variable used to characterize animal obesity. In addition, the misclassification probabilities in animals submitted to normocaloric diet were also evaluated.

\section{MATERIALS AND METHODS}

\section{Animals and experimental model}

This study was performed on thirty-day-old male Wistar rats, supplied by São Paulo State University Animal Center - UNESP Botucatu/SP. Rats were randomly distributed into two groups: normal diet $(\mathrm{ND} ; \mathrm{n}=31)$ and hypercaloric diet (HD; $\mathrm{n}=31)$. ND animals received commercial Labina rat feed (Purina", Paulínia, SP, Brazil) and HD animals were submitted to cycles of five hypercaloric diets $(\mathrm{H} 1, \mathrm{H} 2$, $\mathrm{H} 3, \mathrm{H} 4$, and H5) offered for seven days. Rats were maintained on this dietary regimen for 14 weeks and then sacrificed. The hypercaloric diet was standardized in our laboratory and was utilized for the first time. The groups received water ad libitum. Food consumption was controlled every day, and water was controlled on Mondays, Wednesdays and Fridays. The body weight and body length were measured and used to determine the following parameters: body mass index [BMI; body weight $(\mathrm{g}) /$ lenght $^{2}$ $\left(\mathrm{cm}^{2}\right)$ ]; Lee index [cube root of body weight $(\mathrm{g}) /$ nose-to-anus length $(\mathrm{cm})]$ (Bernardis 1970); body weight to length [FBW/length; weight $(\mathrm{g}) /$ nose-to-anus length $(\mathrm{cm})]$. Serum leptin (ND; $n=13 ; H D=13)$ were measured by the Elisa method using assay kits from Linco Research Inc (Missouri, USA). Rats were housed collectively with four animals per cage, in a temperature controlled $\left(24 \pm 2^{\circ} \mathrm{C}\right)$ environment on a 12 $12 \mathrm{~h}$ light-dark cycle. The study protocol was approved by the Botucatu School of Medicine Research Ethics Committee UNESP and followed the Guide for Care and Use of Experimental Animals.

\section{Hypercaloric Diet Components}

The experimental diets followed specifications from Nutrient Requirements of the Laboratory Rat, as recommended by the National Academy of Sciences. Hypercaloric diets $1,2,4, \& 5$ were designed in our laboratory and contained powdered commercial Labina rat chow (Purina", Paulínia, SP, Brazil), industrialized feeds, protein supplement, vitamins, and minerals (adapted Estadella et al. 2004). The ingredients were first ground and then mixed with the vitamins and minerals. The mixture was then made into pellets, dried in a ventilated drying oven at $55 \pm 5^{\circ} \mathrm{C}$, and stored at $-20^{\circ} \mathrm{C}$. Diet $\mathrm{H} 3$ was commercial Labina with $300 \mathrm{~g} / \mathrm{L}$ sugar added to drinking water. The ingredients ( $\mathrm{g}$ / $\mathrm{Kg}$ ) used to prepare the hypercaloric diets were: H1: ground Labina, 355; roasted peanuts, 176; casein, 123; corn oil, 82; chocolate, 88; corn biscuits, 176; and vitamins and minerals; H2: ground Labina, 439; roasted peanuts, 218; casein, 129; corn oil, 61; French fried potatoes, 153; and vitamins and minerals; H4: ground Labina, 371; roasted peanuts, 185 ; casein, 99 ; corn oil, 68; instant noodles, 185; grated cheese, 92; and vitamins and minerals; and H5: ground Labina, 359; roasted peanuts, 179; casein 105; corn oil, 80; condensed milk, 161; wafer biscuit, 116; and vitamins and minerals. The dietary nutritional composition was analysed by the Animal 
Nutrition and Improvement Laboratory, School of Agronomy Sciences, UNESP Botucatu/SP. The results are shown in Table 1.

\section{Body composition}

Carcass water, fat, and protein content were determined. The percentage of water was calculated as the difference between preand post-drying carcass weight by PPr-PP/ $\operatorname{PPr} \times 100 \%$, where $\operatorname{PPr}=$ pre-drying weight, and $\mathrm{PP}=$ post-drying weight. Bodies were dried at $100 \pm 5^{\circ} \mathrm{C}$ for $72 \mathrm{~h}$ in a ventilated Fanem ${ }^{\circledR}$ oven. After drying, each carcass was wrapped in a paper filter and fat was extracted using a Soxhlet extractor (Corning Incorporated, USA). The percentage of carcass fat was calculated by PP-PSSG/PPr x 100\%, where PP= postdrying weight, $\mathrm{PSSG}=$ dry weight after fat extraction, and $\mathrm{PPr}=$ pre-drying weight. After drying and fat extraction, the carcass was homogenized and nitrogen quantified by the micro-Kjeldahl method (Kjedahl 1965). Protein was determined by \%nitrogen x PSSG x 6.25/100, where $\mathrm{PSSG}=$ dry weight after fat extraction.

\section{Probabilities of misclassication}

The following criteria was used to determine the probabilities of misclassification in variables utilized for identifying obese animals, such as body weight, carcass fat, final body weight to length ratio, Lee index and body mass index: 1) $95 \%$ confidence intervals and upper (UL) and lower (LL) limits were determined from the mean value of variables cited above for both groups; 2) Then, mid-points were calculated between the UL of normocaloric group and LL of the hypercaloric group. This mid-point (SP, separation point) was used to differentiate into which group, obese or lean, the rats would be classified; 3) To classify the animals in one of these groups, the following situation was considered: each animal from the hypercaloric diet group positioned above the SP was considered obese and each rat submitted to the normocaloric diet positioned below the SP was considered lean; 4) The probabilities of classification error were then calculated for each variable analysed. The HD animals below SP were considered obesity-resistant hypercaloric animals (ORH) and ND rats above SP as obesity-prone normocaloric animals (OPN). The best variable to allocate animals to their real group, obese or lean, was the variable that presented minor medium error (probabilities of OPN $+\mathrm{ORH} / 2$ ).

\section{Statistical analysis}

The results of general characteristics were presented as mean \pm standard and submitted to the Student's t-test. The probabilities of

TABLE 1

Composition of normal and hypercaloric diets

\begin{tabular}{lccccc}
\hline & \multicolumn{5}{c}{ Diets } \\
\cline { 2 - 5 } Components & Normal & H1 & H2 & H4 & H5 \\
\hline Protein (\%) & 26 & 27 & 28 & 28 & 26 \\
Carbohydrates (\%) & 54 & 43 & 36 & 33 & 43 \\
Fat $(\%)$ & 3 & 20 & 23 & 13 & 15 \\
Others $(\%) *$ & 17 & 10 & 4.6 & 4.6 & 11 \\
Calories (Kcal/g) & 3.5 & 4.6 & & 4.6 \\
\hline
\end{tabular}

Note: Diet H3 is not shown above because it consisted of Normal Labina diet (Purina, Paulínia, SP) plus sugared water at a concentration of $300 \mathrm{~g} / \mathrm{L}(1.2 \mathrm{kcal} / \mathrm{ml}$ water $)$. H1, H2, H4, \& H5, hypercaloric diets.

* Others: vitamins, minerals, cinders and water. 
misclassification were calculated considering a normal distribution curve, mean, standard deviation and separation points between the groups. Correlations analyses were determined by the Pearson's linear correlation test. The significance level used was $5 \%$.

\section{RESULTS}

\section{General characteristics and correlations}

HD animals ingested less food than ND animals $(\mathrm{ND}=24.4 \pm 2.2$ and $\mathrm{HD}=$ $17.6 \pm 1.7 \mathrm{~g}, \mathrm{p}<0.001)$. Final body weight $(\mathrm{ND}=501 \pm 44$ and $\mathrm{HD}=578 \pm 52$, $\mathrm{p}<0,001)$, final body weight to length ratio $(\mathrm{ND}=18.6 \pm 1.5$ and $\mathrm{HD}=20.4 \pm 1.4$, $\mathrm{p}<0,001)$, Lee index $(\mathrm{ND}=2.65 \pm 0.07$ and $\mathrm{HD}=2.73 \pm 0.06, \mathrm{p}<0,001)$ and leptin $(\mathrm{ND}=2.31 \pm 0.53$ and $\mathrm{HD}=4.82 \pm 1.34$, $\mathrm{p}<0,001)$ were higher in HD than ND animals. Absolute carcass water $(\mathrm{ND}=$ $225 \pm 22$ and $H D=249 \pm 26 \mathrm{~g}, \mathrm{p}<0.001)$, relative $(\mathrm{ND}=18.0 \pm 4.2$ and $\mathrm{HD}=$ $25.5 \pm 4.5 \%, \mathrm{p}<0.001)$ and absolute carcass fat $(\mathrm{ND}=71 \pm 20$ and $\mathrm{HD}=121 \pm 31 \mathrm{~g}$, $\mathrm{p}<0.001)$ were higher in the HD than the
ND group. Relative carcass water $(\mathrm{ND}=$ $57.6 \pm 3.3$ and $\mathrm{HD}=53.0 \pm 3.4 \%, \mathrm{p}<0.001)$ and relative carcass protein $(\mathrm{ND}=16.5 \pm 1.3$ and $\mathrm{HD}=14.2 \pm 1.1 \%, \mathrm{p}<0.001)$ were less in the HD than the ND group. No significant difference was observed in the body mass index (Table 2).

Table 3 shows the results of Pearson's linear correlation test. There were positive correlations between relative carcass fat and final body weight $(\mathrm{r}=0.713 ; \mathrm{p}<0.001)$, body weight to length $(\mathrm{r}=0.645 ; \mathrm{p}<0.001)$, Lee index $(\mathrm{r}=0.642 ; \mathrm{p}<0.001)$, body mass index $\quad(\mathrm{r}=0.347 ; \mathrm{p}<0.01)$ and leptin $(\mathrm{r}=0.802 ; \mathrm{p}<0.001)$.

\section{Probabilities of misclassification}

Table 4 shows classification error probabilities for relative carcass fat, body weight, body weight to length ratio, Lee index and body mass index. Relative carcass fat showed the lowest probabilities of obesity resistant hypercaloric (19.49\%) and obesity-prone normocaloric (18.94\%); it was therefore the best variable to classify obese and lean animals. Relative carcass fat was followed by final body weight $(\mathrm{ORH}=$ $22.06 \%$ and $\mathrm{OPN}=20.33 \%$ ), body weight to

TABLE 2

General characteristics of animals

\begin{tabular}{lcc}
\hline & \multicolumn{2}{c}{ Animals } \\
\cline { 2 - 3 } Variables & Normal diet & Hypercaloric diet \\
\hline Food ingestion $(\mathrm{g})$ & $24.4 \pm 2.2$ & $17.6 \pm 1.7^{*}$ \\
Final body weight $(\mathrm{g})$ & $501 \pm 44$ & $578 \pm 52^{*}$ \\
FBW/Length $(\mathrm{g} / \mathrm{cm})$ & $18.6 \pm 1.5$ & $20.4 \pm 1.4^{*}$ \\
Lee index & $2.65 \pm 0,07$ & $2.73 \pm 0,06^{*}$ \\
BMI $\left(\mathrm{g} / \mathrm{cm}^{2}\right)$ & $0.69 \pm 0.06$ & $0.72 \pm 0.07$ \\
Leptin $(\mathrm{ng} / \mathrm{dl})$ & $2.31 \pm 0.53$ & $4.82 \pm 1.34^{*}$ \\
Carcass fat $(\mathrm{g})$ & $71,0 \pm 20,0$ & $121 \pm 31^{*}$ \\
Carcass fat $(\%) * *$ & $18.0 \pm 4.20$ & $25.5 \pm 4.45^{*}$ \\
Carcass protein $(\mathrm{g})$ & $63.9 \pm 4,99$ & $66.6 \pm 7.38$ \\
Carcass protein $(\%)$ & $16.4 \pm 1.37$ & $14.2 \pm 1.14^{*}$ \\
Carcass water $(\mathrm{g})$ & $225 \pm 22$ & $249 \pm 26^{*}$ \\
Carcass water $(\%)$ & $57.6 \pm 3.3$ & $53.0 \pm 3.4 *$ \\
\hline
\end{tabular}

Values expressed as means \pm standard deviation. FBW, final body weight; BMI, body mass index.

${ }^{*} \mathrm{p}<0.05$ versus normal diet, Student $\mathrm{t}$ test.

** Adiposity index. 
length ratio $(\mathrm{ORH}=26.43 \%$ and $\mathrm{OPN}=$ $26.76 \%)$, Lee index $(\mathrm{ORH}=26.10 \%$ and $\mathrm{OPN}=27.76 \%$ ) and body mass index $(\mathrm{ORH}=40.52 \%$ and $\mathrm{OPN}=41.30 \%)$. In relation to total probabilities of obesityresistant hypercaloric and obesity-prone normocaloric, relative carcass fat showed the lowest medium error (19.21\%); therefore, it was the best variable to separate lean and obese groups. It was followed by final body weight $(21.19 \%)$, body weight to length ratio $(26.59 \%)$, Lee index $(26.93 \%)$ and body mass index $(40.91 \%)$.

\section{DISCUSSION}

The results of this study show that the hypercaloric pellet-diet cycle promoted obesity in Wistar rats, characterized by an increased adiposity index (relative carcass fat) in association with increased body weight, body weight to length ratio, Lee index, and serum leptin. The hypercaloric diet model used in this study, basically increasing fat levels, has no parallel in literature, since we did not find any studies using a rotation cycle of five different types of diet. Dietary manipulation used to induce experimental obesity is presented as pellets or foods in natura, the second better known as cafeteria diets (Sclafani \& Springer 1976). These latter types of diet are highly energetic, very tasty, contain different shapes and are much closer to regular human food. However, they have some restrictions: they are offered in natura, can deteriorate, and it is difficult to quantify

TABLE 3

Correlations between relative carcass fat and body weight, body weight to length, Lee index, body mass index and leptin

\begin{tabular}{lcc}
\hline & \multicolumn{2}{c}{ \% carcass fat } \\
\cline { 3 - 3 } Variables & $\mathrm{R}$ & P value \\
\hline Final body weight & 0.713 & $<0.001$ \\
FBW/Length & 0.645 & $<0.001$ \\
Lee index & 0.642 & $<0.001$ \\
BMI & 0.347 & $<0.01$ \\
Leptin & 0.802 & $<0.001$ \\
\hline
\end{tabular}

FBW, final body weight; BMI, body mass index.

TABLE 4

Probabilities of misclassification

\begin{tabular}{lccc}
\hline \multirow{2}{*}{ Variables } & \multicolumn{3}{c}{ Probability (\%) } \\
\cline { 2 - 4 } Relative carcass fat** & Obesity-prone normocaloric & Obesity-resistant hypercaloric & Medium error \\
FBW & 18.94 & 19.49 & 19.21 \\
FBW/Length & 20.33 & 22.06 & 21.19 \\
Lee index & 26.76 & 26.43 & 26.59 \\
BMI & 27.76 & 26.10 & 26.93 \\
\hline
\end{tabular}

FBW, final body weight; BMI, body mass index.

** Adiposity index. 
how much food animals consume. The dietary model used in this experiment tries to minimize the cafeteria diet effect. Food pellets facilitated accurate measurement of consumption, and helped maintain food integrity and nutrient composition analysis. Food rotation was intended to alter palatability and, consequently, increase food ingestion and body weight gain. However, the results of this experiment showed that the HD group ingested less food than the ND group. In contrast, previous studies showed that hyperlipid diets cause less satiation and consequently higher food ingestion (Dourmashkin et al.; Green et al.; Blundell et al.). Although the results showed absence of excess food consumption, body weight increased in HD rats. The increase of body weight was a consequence of body fat storage enhancement as confirmed by carcass analysis (Table 2). This excessive accumulated adipose tissue has been associated with numerous markers of obesity, such as body weight (Woods et al.), body weight to length ratio (Akiyama et al.), Lee index (Diniz et al.), and serum leptin (Relling et al.), which is produced by adipose tissue in proportion to fat stores (Frederich et al.).

Although our study confirmed the results mentioned above and showed that there were positive correlations between carcass fat and others markers of obesity (Table 3), an important finding of this investigation was that classification error probably occurs in dietary models (Table 4). This misclassification depends on the variables used to classify the groups as obese or lean and may occur in both groups. When relative carcass fat was used, the probability of an animal submitted to hypercaloric diet to be classified as obese when presenting characteristics of a lean animal was $19.49 \%$. However, when body weight was utilized, the misclassification probability was $22.06 \%$ and it may be higher when other markers of obesity are used (Table 4). In relation to obesity-prone normocaloric rats, i.e., animals exposed to normocaloric diet being classified as lean when they truly respond as obese animals ranged from 18.94 to $41.30 \%$.
Therefore, the results of this study showed that animals submitted to different diet models do not always present the expected response. This fact can lead to erroneous animal classification and, consequently, false conclusions. However, this error probability may be minimized by using the best variable. Since studies that evaluate error probabilities in diet-induced obesity were not found, the data this study present can help define the number of animals to be utilized in experimental groups.

In summary, the results of this experiment show that the dietary model used in this study promoted obesity in Wistar rats. However, misclassification probabilities occur when this dietary model is utilized. This misjudgement ranges from $19.49 \%$ to $40.42 \%$ in hypercaloric diet and $18.94 \%$ to $41.30 \%$ in normocaloric diet. The best variable to indicate obesity in experimental models is relative carcass fat. Other markers of obesity may predict excessive accumulate adipose tissue, but may underestimate animal obesity levels.

\section{ACKNOWLEDGMENTS}

The authors thank José Carlos Georgete, Mário Baptista Bruno, Sandra Fábio, Adriana Ap. Interdonato Passaroni and Colin Edward Knaggs for technical assistance. This paper was supported by the Research Support Group from Botucatu School of Medicine and Fundunesp, São Paulo - Brazil.

\section{REFERENCES}

1. AKIYAMA T, TACHIBANA I, SHIROHARA H, WATANABE N, OTSUKI M (1996) High-fat hypercaloric diet induces obesity, glucose intolerance and hyperlipidemia in normal adult male wistar rat. Diab Res Clin Pract 31: 27-35

2. ASTRUP A, BUEMANN B, WESTERN P, TOUBRO S RABEN A (1994) Obesity as an adaptation to a highfat diet: evidence from a cross-sectional study. Am J Clin Nutr 59: 350-5

3. BERNARDIS LL (1970) Prediction of carcass fat, water and lean body mass from Lee's nutritive ratio in rats with hypothalamic obesity. Experientia 26: 789-90

4. BLUNDELL JE, BURLEY VJ, COTTON JR, LAWTON CL (1993) Dietary fat and the control of energy intake: 
evaluating the effects of fat on meal size and postmeal satiety. Am J Clin Nutr 57: 777-8

5. DINIZ YS, BURNEIKO RM, SEIVA FR, ALMEIDA FQ, GALHARDI CM, FILHO JL, MANI F, NOVELLI EL (2008) Diet compounds, glycemic index and obesity-related cardiac effects. Int J Cardiol 124: 92-9

6. DOURMASHKIN JT, CHANG GQ, GAYLES EC, HILL JO, FRIED SK, JULIEN C, LEIBOWITZ SF (2005) Different forms of obesity as a function of diet composition. Int J Obes Rel Met Disord 9: 1-11

7. ESTADELLA D, OYAMA LM, DAMASO AR, RIBEIRO EB, NASCIMENTO CMO (2004) Effects of palatable hyperlipidic diet on lipid metabolism of sedentary and exercised rats. Nutrition 20: 218-24

8. FREDERICH RC, HAMANN A, ANDERSON S (1995) Leptin levels reflect body lipid content in mice: evidence for diet-induced resistance to leptin action. Nat Med 1: 1311-1314

9. GREEN SM, BURLEY VJ, BLUNDELL JE (1994) Effect of fat and sucrose containing foods on the size of eating episodes and energy intake in lean males: potential for causing overconsumption. Eur J Clin Nutr 48: $547-55$

10. JANG I, HWANG D, LEE J, CHAE, K, KIM Y, KANG, T (2003) Physiological difference between dietary obesity-susceptible and obesity resistant Sprague Dawley rats in response to moderate high fat diet. Exp Anim 52: 99-107

11. KJELDAHL (1965) Official methods of analysis. $8^{\text {th }}$ ed. Washington: Association of Official e Agriculture Chemists
12. LEVIN BE, DUNN-MEYNELL AA, MCMINN JE, ALPEROVICH M, CUNNINGHAM-BUSSEL A, CHUA JR SC (2003) A new obesity-prone, glucoseintolerant rat strain (F.DIO). Am J Physiol Regul Integr Comp Physiol 285: 1184-91

13. LISSNER L, HEITMANN BL (1995) Dietary fat and obesity: evidence from epidemiology. Eur J Clin Nutr 49: 79-90

14. RATTIGAN S, CLARK MG (1984) Effect of sucrose solution drinking option on the development of obesity in rats. J Nutr 114: 1971-7

15. RELLING DP, ESBERG LB, FANG CX, JOHNSON WT, MURPHY EJ, CARLSON EC, SAARI JT, REN J (2006) High-fat diet-induced juvenile obesity leads to cardiomyocyte dysfunction and upregulation of foxo3a transcription factor independent of lipotoxicity and apoptosis. J Hypertens 24: 549-561

16. SCLAFANI A, SPRINGER D (1976) Dietary obesity in adults rats: similarities to hypothalamic and human obesity syndromes. Physiol Behav 17: 461-71

17. THIBAULT L, WOODS SC, WESTERTERPPLANTENGA MS (2004) The utility of models of human energy homeostasis. Br J Nutr 92: 41-5

18. WOODS SC, SEELEY RJ, RUSHING PA, D'ALESSIO D, TSO P (2003) A controlled high-fat diet induces an obese syndrome in rats. J Nutr 133: 1081-7

19. WORLD HEALTH ORGANIZATION - WHO [homepage on the Internet]. Obesity and overweight. Geneva; 2004. [cited 2004 may 20]. Available from: http: // www.who.int. /dietphysicalactivity/ 
